\title{
Distributed quantitative evaluation of 3D patient specific arterial models
}

\author{
A. Giachetti \\ M. Tuveri \\ G. Zanetti \\ CRS4 - VI Strada Ovest, \\ Z.I. Macchiareddu, Uta(CA), Italy \\ e-mail \{giach,mtuveri,zag\}@ crs4.it
}

\begin{abstract}
In this paper we describe a new system for the $3 D$ reconstruction and distribution on the net of models for vessels structures. The system is specifically designed to support measurements of medical interest. We describe $2 D$ and $3 D$ segmentation methods implemented and the procedure used to build interactive VRML97 models. The experimental section presents a comparison between segmentation methods, and a first application to surgical planning for endovascular repair of Abdominal Aortic Aneurysms.
\end{abstract}

\section{Introduction}

In the near future a large amount of medical data will be transmitted across Internet for remote diagnosis, electronic patient records retrieval, home care, etc.. Images, annotations and texts can be easily transmitted with standard protocols and can be viewed by medical doctors everywhere by using common web browsers. Many examples of web based telemedicine have been proposed and presented in conference papers $[2,3,4]$. Moreover, there are few examples in literature relative to the web distribution of $3 \mathrm{D}$ models of organs, and they are not usually tailored to real clinical needs or diagnostic purposes. Typically, these models are used only for qualitative study or to implement simple surgical simulators [8], albeit current technology limitations force a strong simplification of the procedures being simulated. In this paper, we show that it is possible to use current web technology standards, i.e., VRML, to assemble patient specific models of vessels that allow surgeons to measure inherently $3 \mathrm{D}$ quantities that are important, for example, to surgical planning, e.g., the angles between vessels at the iliac bifurcation. The models are reasonably compact in size, with an average size of few hundred kilobytes, and can be quickly transferred across Internet. The data sent is essentially the vessel surface geometry plus embedded code and pre-computed tables to support the measurements. More- over, the model is structured so that by selecting any point on a centerline it is possible to require, to a dedicated web server, a slice across the original volumetric dataset(s) orthogonal to the local direction of the centerline, thus allowing the doctor to consult at the same time the original medical imaging data. The paper is organized as follows: Section 2 will describe different methods for 3D segmentation, Section 3 will describe the VRML generation, Section 4 will present the application to Abdominal Aortic Aneurysm endovascular treatment, Section 5 will present experimental results.

\section{Segmentation and 3D reconstruction}

\subsection{The arterial tree data structure}

In order to perform measurements on vascular geometries (and also to support other virtual reality and numerical simulation applications) we designed a specialized data structure that we named "Arterial Tree". An Arterial Tree is defined as the union of a complete surface mesh describing the vessels internal surface, and a skeleton joining series of 1D lines representing the vessels centerline. This data structure allows us to

- realize precise measurements of vessel length;

- simplify the estimate of a plane perpendicular to the vessel;

- provide a geometrical model that can be used to build a volume mesh inside the vessel for numerical simulation of blood flows [1].

We implemented three methods for the reconstruction of the AT structure and developed an user friendly interface to control the use of these methods and other image processing tools (see Fig.1).

Methods are: 
- A: Segmentation from 2D contours: The dataset is cut with series of planes approximately directed along the vessel branches directions. Contours are extracted with snake balloons $[13,14]$ and joined in simple tubes. Finally, tubes are glued together to build the complete tree. The centerline is built by generating a spline passing through the centers of mass of the extracted contours.

- B: Segmentation from isosurfaces and automatic centerline extraction: Thresholding and marching cubes [20] are used to compute the vessel surface, while the centerline is automatically extracted with an algorithm initialized giving a point inside the vessel and described in section 2.3.

- C: Segmentation from Simplex Balloon and automatic centerline extraction: Simplex balloons [15] are a deformable surfaces in the 3D space. Initializing surfaces as small spheres inside the vessels and choosing the correct forces inflating and driving surface points to the vessel border, we can obtain a smooth vessel surface. The same algorithm as in method B is used for the centerline extraction.

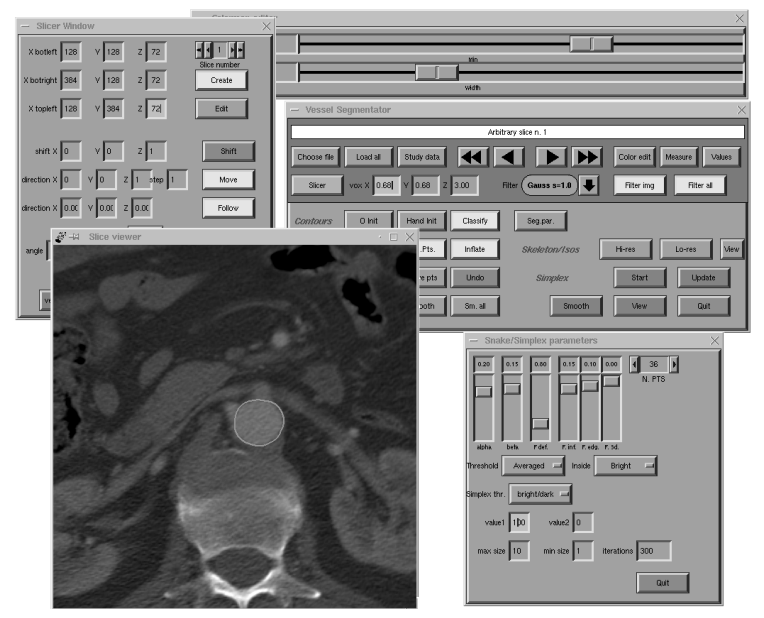

Figure 1. The user interface allowing the easy use and tuning of all the segmentation algorithms implemented.

We now discuss in more detail the three segmentation methods; their performances on a specific application will be discussed in the experimental section.

\subsection{Method A: Segmentation from 2D slices}

The basic segmentation procedure is summarized in Fig. 2. Series of contours with a fixed number of points are extracted on slices with arbitrary position and orientation in the 3D dataset. Images are computed from the 3D dataset using tri-linear interpolation.

The user interface include a menu to select the slices from the dataset and give the possibility of extracting contours with a a simple region growing, or as default choice, with an elastic contour or "snake". Snakes are elastic contours that undergoes a "physical" evolution driven by "forces". A contour is defined as a set of connected points subjected by external force fields and interacting with their neighbors. If the forces are chosen correctly, the contour evolution is stopped near the desired image feature. The initial contour can be chosen as:

- a small circle around a selected point,

- the contour detected on the previous slice (useful choice to segment vessels)

- by drawing the approximate position by hand.

Usually the first option is used for the starting slice of a series, the second for the other slices, and the third in case of noise or problems in automatic detection. The evolution of our snakes is driven by standard elastic and rigid forces depending on the derivatives of the contour; by an inflating force, like in [14]; by an edge attraction and by a deflating term that makes the contour shrink along the normal vector direction if the image brightness is above (or under) a local threshold. The threshold can be selected close to the Hounsfield value (i.e. the material density) corresponding to the contrast medium. However, for some of the data analyzed, the contrast was not always perfect and in this case it can be a better choice to use an adaptive algorithm that searches for strong discontinuities along the directions perpendicular to the contour and selects the corresponding "external" value as threshold.

The major advantage of the contour based approach is that it is immediately visually clear if the algorithm worked well. The user interface provides of several post processing facilities to modify the result if necessary:

- Some points can be moved to a different location.

- Contours can be smoothed with a center based algorithm as follows: first one computes the local average distance of the point and two neighbors from the contour center and then the point is shifted in order to have the distance from the center equal to that value. This can be really effective if the contour is not too irregular.

- The number of points can be changed and contours can be re-sampled making the distance between successive 
points approximately constant. This is done calculating the length of a spline joining the points, the corresponding average point spacing and finally replacing the points along the line.

- Selected points can be marked as "Fixed", and these points are not moved during further application of the snake algorithm.

These manual processing are sometimes necessary if the signal to noise ratio in the images is low, and for example when calcifications are clearly visible but hard to discriminate with automatic methods.

When the segmentation of a contour series is finished, the user saves all the contours points in a file, then resets the slicer tool and can extract a new series of contour, corresponding to another vessel branch, until all the geometries of his interest are reconstructed.

The second step in the geometry building is the generation of vessel segments. Being each series composed of the same number of contours, evenly spaced, the program automatically finds the correspondence between points of successive contours, and connect with an edge corresponding point and each point with a neighbor of the corresponding point, building a triangulated surface. If smaller triangles are required, the algorithm can add nodes. Finally the vessel centerline is built as a spline connecting the center of mass of the contours.

Once vessel segments are built from the contour series, they are finally glued together with an anastomosis operation, i.e. the insertion of the secondary branch into the largest one. It is important, therefore, that the intersection of each segment and one of the others is a closed contour, but this can be obtained easily by checking the contour positions on the slicer window. This is done as follows: first the intersection between the two external surfaces is found, then the part of surface inside the largest vessel is removed and a new triangulation is created near the intersection curve.

Errors introduced in the geometry depend on the number of slices used for the segmentation, if the slices are sufficiently close each other, the error can be considered equal to the voxel dimension. The computed centerline can be exploited for an iterative refinement of the geometry: first new cutting planes orthogonal to the centerline are generated, then new contours are extracted (Fig 3). Geometrical operations are realized with the support of the XOX Shapes MicroTopology libraries [19]. The choice of the geometrical engine, however, is not critical, and we are testing other methods to obtain the results.

\subsection{Automatic centerline extraction}

Methods B and C are based on surface extraction with Marching Cubes or deformable surfaces. In both cases, to
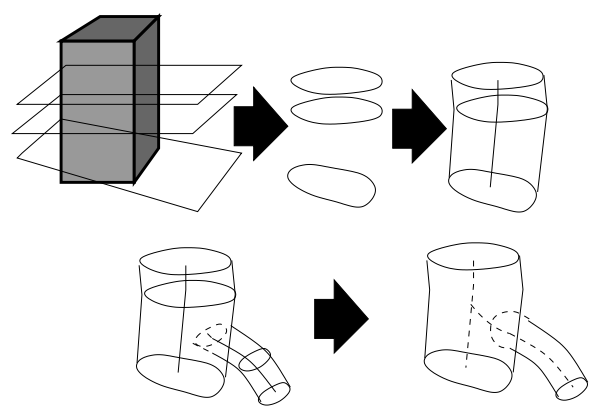

Figure 2. Arterial Tree building from contours and centerlines. Several contour series can be extracted and several vessel segments, with an external surface and a center line are consequently built. Finally they are joined in an unique tree, with only one triangulated surface and a tree-shaped centerline.

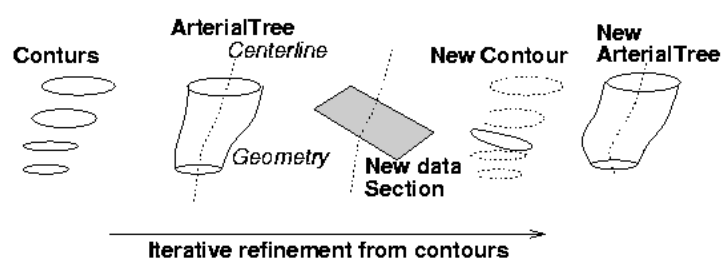

Figure 3. Arterial segments can be refined adding new planes orthogonal to the computed centerline.

build the AT structure, we have also to find the centerline of the vessel. We therefore have implemented an algorithm for the centerline extraction directly working on the voxel data, that require the user just to select on the first slice a pixel inside the vessel lumen and a threshold value. Then the algorithm, derived from the voxel coding technique introduced in [16], works as follows:

- Compute a "distance" of each internal point from the connected region boundaries (Boundary seeded distance, BSD).

- Compute a "distance" of each internal point from the starting point of the segmentation or "seed point" (SSD).

- Find maximum of the SSD.

- Starting from the point corresponding to this maximum, create a chain connecting at each step the last detected point with the neighboring voxel with the lowest value of SSD. The chain must end at the seed point. 
- For each point of the chain find the connected region with the same SSD. Find the maximum of the BSD in the region and move the chain point there. Put to zero the SSD value of the region. The resulting chain is a vessel centerline branch.

- Find the maximum of the changed SSD map and repeat the same procedure, stopping the chain detection when a voxel with zero SSD is found. The result is a new vessel branch. Repeat the procedure until the SSD is not cleared.

- Branches are finally smoothed and connected.

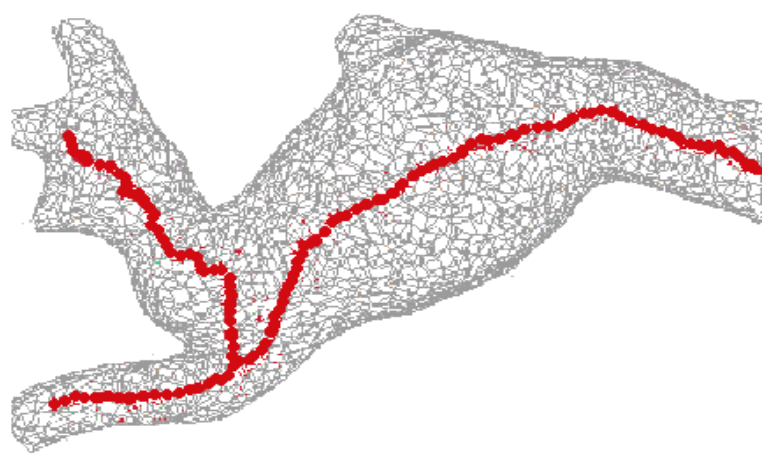

Figure 4. Balloon segmentation of an iliac bifurcation with the corresponding skeleton extracted inside.

\subsection{Method B: isosurface extraction}

The second option available to the user is the extraction of an isosurface. After a Gaussian or median smoothing, the dataset is binarized and a region growing algorithm is started from the seed given by the user. Finally the marching cubes algorithm [20] is applied.

\subsection{Method C: 3D balloon reconstruction}

Deformable surfaces can now be used for 3D segmentation due to the improved computational power of PC's and workstations. A survey on their use can be found in [21]. Specific applications of have already be presented by other authors [22], who used also some model based constraint to segment the correct structures. The method here used is based on the simplex mesh geometry introduced by Delingette [15]. As defined in the paper, the generic Simplex Mesh is a $\mathrm{N}$ dimensional mesh with $\mathrm{N}+1$ connectivity. The simplex mesh we use is therefore a closed surface mesh composed by nodes each connected with three neighbors.

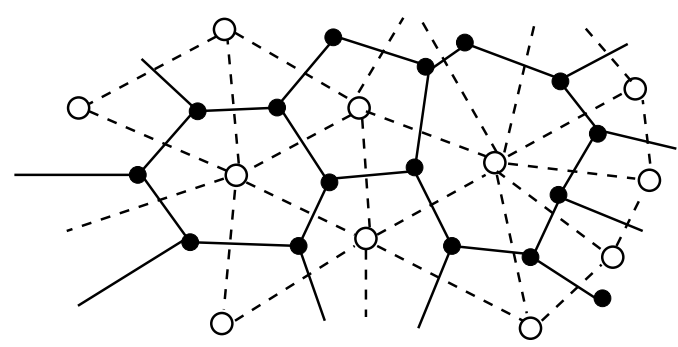

Figure 5. A simplex mesh (black nodes) and its dual triangulation (white nodes)

We make the nodes move under the influence of an inflating force directed along the surface normal, an elastic smoothing force ("surface orientation continuity constraint") and two image forces: the deflating one compensating the inflating one where the local average of the gray level differs from the internal value more than a fixed threshold and an edge attraction. Controls on maximum and minimum of the face size have been introduced to have the desired mesh refinement. We used also the methods to collapse or divide faces of the mesh well described in [15]. The final mesh is converted in the "Dual" form (i.e. a new mesh with nodes in the center of the simplex faces and connections corresponding to the simplex edges, see Fig5), in order to have a smooth triangulation to be rendered.

The simplex mesh, in fact, due to its definition, is in general composed by polygons that are not necessarily planar, and therefore cannot be easily represented.

In order to use this method, the user must click on a point inside the vessel lumen. The surface is then initialized as a small sphere and is inflated until the surface is not blocked by edges or changed image value. The user can control the maximum number of iterations to be performed, force parameters and the maximum and minimum size of the polygons.

\section{VRML97 measurable models of vessel}

VRML97 is a powerful language to describe 3D scenes and it is the standard language for Virtual Reality on the web. VRML97 plug-ins for Internet Explorer and Netscape Communicator (i.e. Cosmo Player (www.cai.com/cosmo) or Cortona (www.parallelgraphics.com)) are available on the net and can be downloaded at no charge. We tested our code with Cosmo Player 2.1 for Microsoft Windows 98/NT both with Netscape and Internet Explorer. Using a perl script developed for the purpose, we automatically convert models of arterial trees representing abdominal aortic aneurysms into VRML97 files with hidden ECMAScript code enabling the user to use the browser to perform 


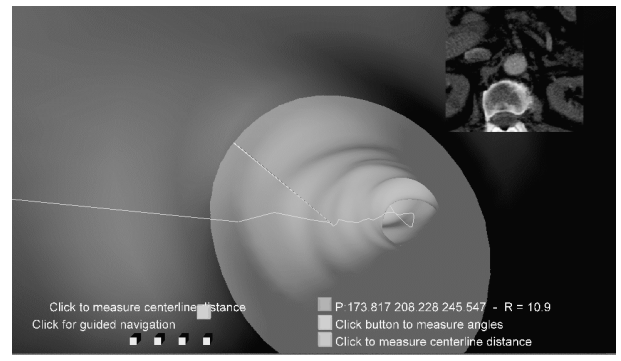

Here are some details about our data conversion: VRML97 worlds are generated automatically from the Arterial Trees structure. Different "nodes" of VRML97 have been used to represent the geometry of the vessel surface (IndexedFaceSet) and the vessel centerline (IndexedLineSet); The centerline is also duplicated alone in another part of the scene alone to make easier the measurement of angles and distances (Fig. 7,8). The Viewpoint node is used to define a set of privileged points of view (at the end of each vessel branch and an external global view). The TouchSensor node have been used to let the user interact with the geometries; we exploited it not only to get the coordinates of the vessel, but also to create buttons to select the possible actions to be done. The Timer node have been also used to create animations because it changes the value of an output variable as defined by the programmer. Routing the output of a Timer to other nodes called PositionInterpolator and OrientationInterpolator that give, as suggested by the name, a series of values of coordinate and direction, we can control the the motion of the point of view in order to create a driven navigation along a fixed path. Our conversion script creates automatically a guided navigation along each branch of the tree along the vessel centerline.

\subsection{Support for measurements}

The VRML97 language specification includes a particular node, called Script, that is extremely powerful. It makes possible to call a custom ECMAScript or Java routine hidden in the file or saved in a known location after an event on the scene. The routine can take as input values depending on the scene and on the user actions and output values can be routed to the other nodes.

Using ECMA scripting we implemented three methods to measure parameters useful for medical applications.

The first consists of printing the 3D coordinates and the distance from the centerline for each point of the surface when the mouse is clicked on it. A plane perpendicular to the centerline is also shown for an easy control of the distance direction (Fig. 6).

The second consists of measuring the distance of two points on the centerline following the line itself (Fig. 7). This is very important, because usual measurements of vessel length done with 2D imaging or endoscopy are often wrong due to the effect of vessel curvature $[17,11]$.
Figure 6. The VRML97 model can be inspected, navigated and measured from any PC with a web browser and a plug-in. Here a "virtual endoscopy" with radius measurement is shown.

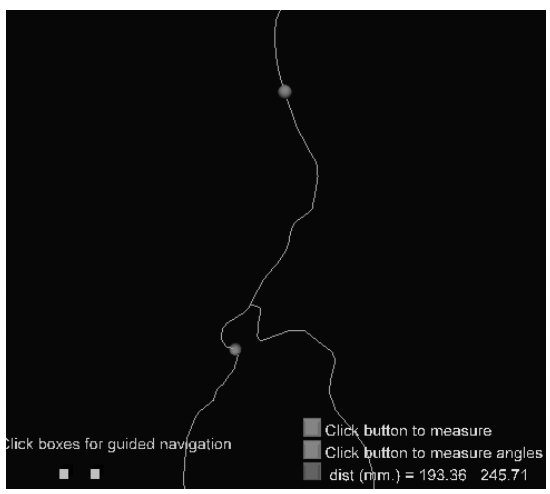

Figure 7. Measurement of centerline distance: the user just clicks on two points on the centerline, and on the browser are automatically displayed the distance between the points and the distance between the points following the centerline, that can be extremely different and it is the really important parameter to evaluate for aneurysm measurements.

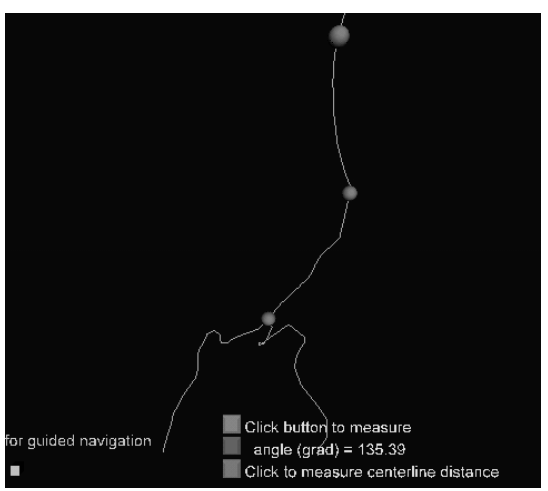

Figure 8. Measurement of centerline angles: the user can place the three reference points just by clicking with the mouse on the centerline, and the angle is automatically shown. 
The last one is the measurements of angles defined by clicking on three points on the centerline (Fig. 8). Furthermore, we added to the scene an image viewer so that when a point on the surface or on the centerline is clicked for measurement, the image defined by the intersection of the plane perpendicular to the centerline and passing through the point clicked and the dataset is automatically shown. Images are created off-line, compressed ad stored in a common directory on the server side. Being very small they can be downloaded quickly. However, it is possible also to change the code to have them pre-loaded on the client side. Another useful tool introduced in the models is an automatic test aimed at checking if a probe of fixed diameter can pass through the lumen, relevant to investigate if a catheter of known size can be introduced in the vessel.

\section{Application: Abdominal Aortic Aneurysms evaluation}

In order to verify the quality and the usefulness of our model reconstructions, we have applied our system to the analysis and the endovascular surgical planning for $\mathrm{Ab}$ dominal Aortic Aneurysms. An abdominal aortic aneurysm (AAA) is a bulge in the aorta in the abdomen [17, 18]. AAA is a vascular disease with life-threatening implications, that is becoming increasingly common in aging populations. When the risk of rupture is high, a surgical intervention is required to repair the aorta. The standard approach is the arterial grafting in open surgery, but the mortality of this kind of intervention is high. A recently developed alternative to open surgery, is the endovascular repair, consisting in the introduction of a prosthesis with a catheter passing through the iliac artery. This procedure can be performed in a surgical or a radiological suite, but it requires a preliminary accurate assessment of patient's specific anatomy. The measurements of the geometry of the aorta is therefore extremely important. Measurements are usually done on printed 2D slides [25, 18, 23], and this fact can introduce large errors [17, 11].

Using patient specific VRML97 models reconstructed with our method, any user can measure the geometric parameters of interest with good accuracy.

This is particularly interesting: in fact, other computer assisted volumetric approaches have been proposed and tested for the same purpose [17], but they require complex and proprietary software on visualization workstations. Our models allow a simple and fast measurement of the required values without the need of particular hardware or software and the measurement of aortic aneurysms seemed a good test-bed to show the usefulness of the approach chosen. Moreover, this technology is ideally suited to support distributed services that improve the measurement quality (using the $3 \mathrm{D}$ reconstruction) and allow also collaborative sur-
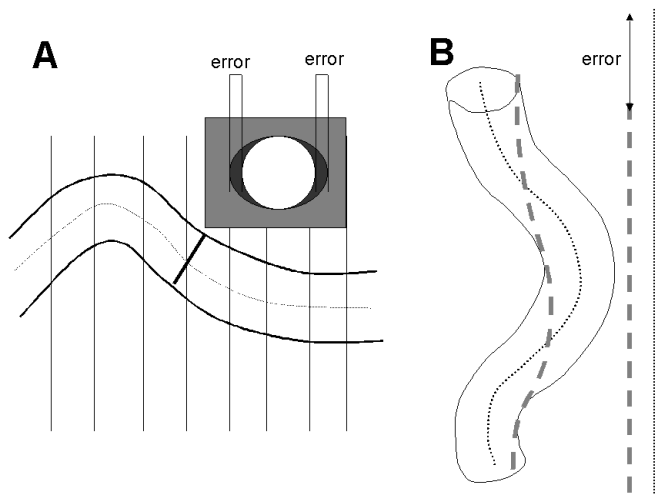

Figure 9. Errors introduced by estimating vessel length and diameter from 2D slices or catheter trajectory. A: the diameter measured from a CT slice can be superestimated is the vessel direction is not perpendicular to the slice plane. B: The vessel length measured from the catheter trajectory is underestimated.

gical planning between remote sites.

\section{Experimental results}

\subsection{Comparison of segmentation techniques}

We tested the three segmentation methods described in Section 2 on different CT data sets coming from the Radiology dept. of the University of Pisa and from the Hospital of Ravenna. On datasets obtained following the standard protocols for abdominal aortic aneurysm repair planning (resolution is less than $2 \mathrm{~mm}$. in the $\mathrm{z}$ direction and contrast liquid is injected before the acquisition so that signal to noise ratio is high) all three methods perform well and the measurements are consistent (see Fig. 11).

To test the robustness of the algorithm, we tested the system also on images with poor contrast and lower values of $\mathrm{z}$ resolution. For the isosurface extraction, a large slice spacing makes difficult to have a smooth surface and even an unique surface for small vessels. A poor signal to noise ratio causes a difficult threshold setting for discriminating the vessel lumen from the background and it makes often not possible to distinguish automatically calcium from contrast. This means that, even using median filtering and morphological closing, we cannot get automatically a good detection of the lumen and, in some cases, we obtain also discontinuous surfaces and false detections of borders.

It must be also considered that local errors are likely to be present in reconstructions. For the contour-based recon- 


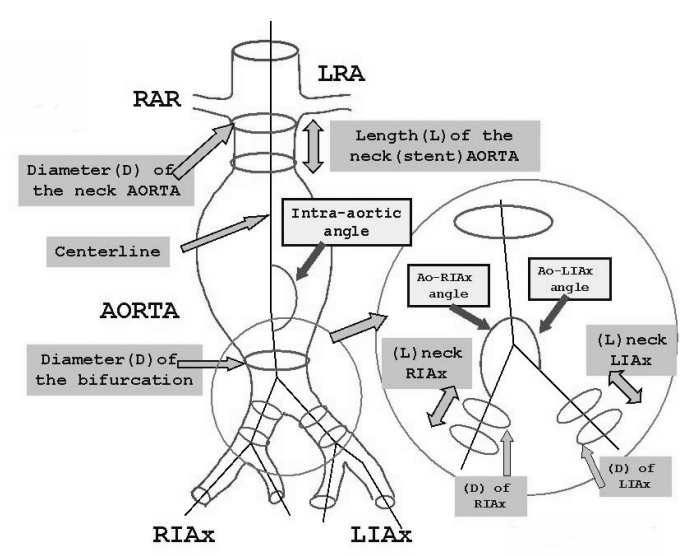

Figure 10. A large number of measurements are necessary, including the length and diameter of the proximal aortic "neck", the length and diameter of the distal "cuffs", and the length of the two graft limbs. Measurements for an endovascular tube graft are only slightly less complex.

structions, errors can be present near bifurcations, even if the image quality is good, due to possible approximation in triangulation methods. 3D balloons are affected by the necessity of finding equilibrium between image based and elastic forces, and can fail in regions where curvature is quite high and signal to noise ratio is not high. These errors do not affect, usually, the measurement of the parameters of surgical interest.

\begin{tabular}{|c|c|c|c|}
\hline Measure & $\mathrm{A}(\mathrm{mm})$. & $\mathrm{B}(\mathrm{mm})$. & $\mathrm{C}(\mathrm{mm})$. \\
\hline Neck r. & $11.8 \pm 0.7$ & $11.9 \pm 0.7$ & $11.4 \pm 0.7$ \\
Max r. & $15.4 \pm 0.7$ & $16.0 \pm 0.7$ & $16.2 \pm 0.7$ \\
Length & $115 \pm 2$ & $115 \pm 2$ & $115 \pm 2$ \\
\hline
\end{tabular}

Table 1. If the image quality is good, measures of parameters performed on the three models with methods A, B and C are compatible. Here are, for example the results of corresponding measurements on the three models of Fig. 11.

\subsection{Sensitivity to initialization and parameters change}

One thing to be considered, when analyzing the results is our methods, as all the segmentation techniques, are influenced by the thresholds chosen and by the parameters
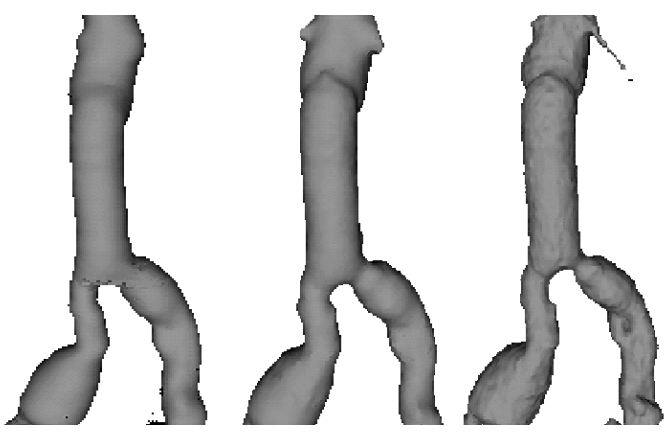

Figure 11. Comparison of the three reconstruction methods on a sufficiently good acquisition. Left: Reconstruction performed through 2D contour extraction. Center: Reconstruction performed using Simplex balloon and automatic centerline extraction. Right: Reconstruction performed using marching cubes and automatic centerline extraction.

used and therefore are user dependent. The results obtained with our methods are strongly dependent on the threshold choice and on the value of elastic parameters. They are not strongly dependent on the initialization point, both for $2 \mathrm{D}$ and 3D methods. Different gray or edge threshold or a different elastic constant can change the segmentation result and thus these parameters should be selected under the supervision of an expert radiologist with a non trivial experience in segmentation, because even if many of the program options are automatic or semi-automatic, the user needs to have a feeling of the algorithms and of images features in order to perform a better reconstruction. This fact suggests that our approach where the 3D reconstruction is outsourced to the centralized reconstruction center is useful.

\subsubsection{Sensitivity issues for 2D methods}

Region growing result depends only on the choice of the threshold, so the selection of a reasonable protocol is sufficient to guarantee user independence. The behavior of a snake can be changed in many ways, acting on elastic parameters, force constants, the number of points chosen, and so on. This means that the segmentation result depends on the user's choices. This is not a drawback: it makes possible, acting on that parameters, to segment different structures on different kind of images. The user dependency can be, however, controlled, by using, for each kind of task required, a detailed protocol giving a parameters set for the contour extraction. The one we are currently using is:

- Perform 3x3 median filtering.

- Select a correct windowing to show the desired struc- 
tures.

- Choose a sufficient number of points in order to have a point spacing of a few pixels.

- Use an image force selecting a bright region in dark background with a fixed threshold and the elastic and force constants saved after tuning for these kind of segmentation.

- Initialize the contour inside the region letting it evolve until the contour motion is stopped.

- Re-sample the contour to have the desired number of points equally spaced.

Once the protocol is defined, our goal is to prove that that the result do not depend on the contour initialization, i.e. once the parameters are set, the method is user-independent. We selected some slices with differently shaped vessel sections and measured the differences between several contours extracted with different initial contour positioning. Results(see Table 2) show clearly that when the contour is well defined, the contour extraction is user-independent, and the differences between the segmentation techniques are not relevant.

Only if the image quality is bad, for example when the contrast is low (Fig. 12) there is no way to use the standard parameters obtaining a user-independent result. The user, in this case, must draw the contour by hand or correct the result where not reliable (for example near plaques or artifacts). This fact underlines the importance of having an experienced person doing the segmentation work, and this should be considered a specialized task even if performed with user friendly software.
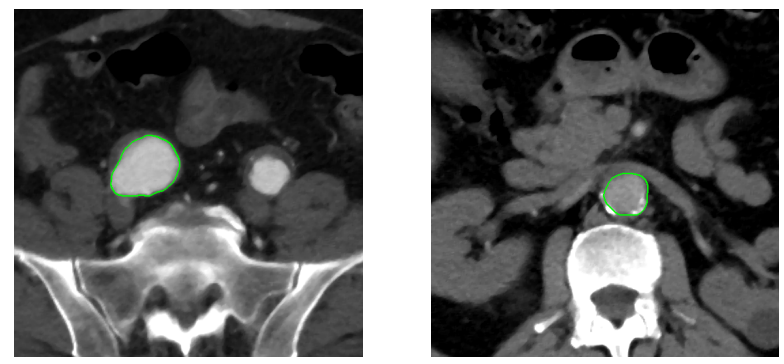

Figure 12. Left: One of the slices use to test the user independence of the system when the image acquisition is correct. Right: One of the slices where the contrast is too poor to have user independent results due to bad image acquisition

\begin{tabular}{|c|c|c|}
\hline Methods & avg.diff. & max.diff. \\
\hline snake/snake & $0.11 \mathrm{~mm}$. & $0.36 \mathrm{~mm}$. \\
reg. grow/snake & 0.14 & 0.44 \\
manual/snake & 0.17 & 1.11 \\
simplex section/snake & 0.23 & 0.64 \\
isosurface sec./snake & 0.33 & 0.97 \\
\hline
\end{tabular}

Table 2. Comparison of segmentation results obtained by different users with different techniques: the table shows the average and maximum distance between points belonging to different snakes segmentation, distances between a contour obtained with region growing and one obtained with snakes, between a contour drawn by hand and a snake segmentation, between snake points and a simplex mesh and between snake points and an extracted isosurface. The pixel size was 0.7 $\mathrm{mm}$. and this means that differences are negligible compared with quantization error.

\subsubsection{Sensitivity issues for 3D methods}

If a fixed threshold is chosen, a comparison between balloon isosurface and manually selected contours or 2D snakes is possible. Fig. 13, for example, shows the nodes of a simplex balloon lying on a CT slice, superimposed to the corresponding gray values and the corresponding 2D snake.

We performed this comparison on all the data sets and we have found that for images acquired using standard protocols the two reconstructions are consistent at the voxel level.

\subsection{Parameter estimation form VRML models}

We have finally taken 12 models of aortic aneurysm reconstructed from CT data provided us by the Radiology dept. of the University of Pisa and from the Hospital of Ravenna, and chosen selected parameters to be measured with a standard 2D technique currently used by surgeons and with our system. The parameters are:

- Diameter of the aortic neck

- Length of the aneurysm

- Intra-aortic angle

- Diameter of the bifurcation

The data selection includes a few acquisitions with poor contrast or resolution. Therefore we decided to adopt as standard segmentation method for the VRML97 generation 


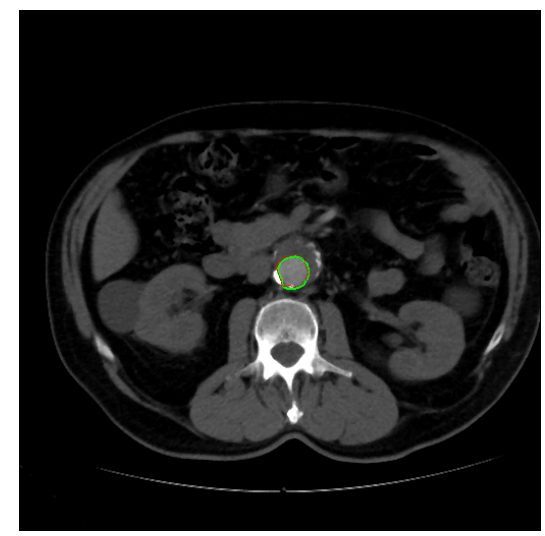

Figure 13. If image quality is good and vessel structure is simple, points from contour segmentation and from a 3d model (in this case Simplex based) sections are correctly superimposed: no relevant differences are visible between the two contours printed over the image

the $2 \mathrm{D}$ contour based segmentation. The comparison has been realized in the following way: for the vessel diameter, the doctor working using the standard measurement protocol on the CT slices measured the minimum of the local vessel radius, the other working on the VRML measured in four selected vessel points at the same $\mathrm{z}$ location, the smallest distance between the point and the centerline. We considered measurement errors equal to the voxel size in $\mathrm{x}, \mathrm{y}$ directions.

The results are good, except for some cases with inconsistencies at the bifurcation (case 2,11). In these cases this is probably due to incorrect estimates made with the "classical" method, due to varying vessel tortuosity. Errors appear to depend not only on the vertical resolution but also on vessel tortuosity. By looking at the reformatted image displayed on the browser it is possible to check the local quality of the reconstruction (see Fig.14).

\section{Discussion}

Computer vision, virtual reality and Web technologies can really have clinically relevant applications. In this paper we presented a novel application using customized image processing technique and web technologies to help surgeons in diagnosis and pre-operative measurements of 3D structures. The application is simply to be considered as an example showing that the accuracy requested by the application is achievable with these methods, but other applications can be obtained easily.

\begin{tabular}{|c|c|c|}
\hline n. & Rad(mm.) & VRML rad.(mm.) \\
\hline 1 & $10.6 \pm 0.7$ & $10.6 \pm 0.7$ \\
2 & $10.4 \pm 0.7$ & $8.9 \pm 0.7$ \\
3 & $16.8 \pm 0.7$ & $17.8 \pm 0.7$ \\
4 & $9.9 \pm 0.7$ & $9.9 \pm 0.7$ \\
5 & $12.5 \pm 0.7$ & $11.8 \pm 0.7$ \\
6 & $11.0 \pm 0.7$ & $11.0 \pm 0.7$ \\
7 & $10.3 \pm 0.7$ & $9.2 \pm 0.7$ \\
8 & $12.3 \pm 0.7$ & $12.5 \pm 0.7$ \\
9 & $10.3 \pm 0.7$ & $9.7 \pm 0.7$ \\
10 & $10.6 \pm 0.7$ & $9.3 \pm 0.7$ \\
11 & $9.7 \pm 0.7$ & $8.9 \pm 0.7$ \\
12 & $10.3 \pm 0.7$ & $10.3 \pm 0.7$ \\
\hline
\end{tabular}

Table 3. Minimum radius of aortic neck (measured carefully at the same $z$ location using a standard $2 \mathrm{~d}$ method, and working directly on the 3D model.

\begin{tabular}{|c|c|c|}
\hline n. & Z distance(mm.) & Length (VRML) (mm.) \\
\hline 1 & $106 \pm 2$ & $115 \pm 2$ \\
2 & $88 \pm 2$ & $94 \pm 2$ \\
3 & $88 \pm 8$ & $101 \pm 8$ \\
4 & $110 \pm 2$ & $120 \pm 2$ \\
5 & $120 \pm 2$ & $137 \pm 2$ \\
6 & $129 \pm 5$ & $166 \pm 5$ \\
7 & $164 \pm 3$ & $170 \pm 3$ \\
8 & $129 \pm 3$ & $146 \pm 3$ \\
9 & $125 \pm 5$ & $148 \pm 5$ \\
10 & $120 \pm 3$ & $133 \pm 3$ \\
11 & $110 \pm 3$ & $133 \pm 3$ \\
12 & $120 \pm 3$ & $127 \pm 3$ \\
\hline
\end{tabular}

Table 4. $Z$ component of aneurysm length and real length measured on the VRML models. 


\begin{tabular}{|c||c|c|}
\hline n. & Min rad.(mm.) & VRML min (mm.) \\
\hline 1 & $9.9 \pm 0.7$ & $10.4 \pm 0.7$ \\
2 & $6.5 \pm 0.7$ & $6.3 \pm 0.7$ \\
3 & $12.1 \pm 0.7$ & $11.3 \pm 0.7$ \\
4 & $15.7 \pm 0.7$ & $15.0 \pm 0.7$ \\
5 & $12.1 \pm 0.7$ & $11.8 \pm 0.7$ \\
6 & $14.0 \pm 0.7$ & $13.5 \pm 0.7$ \\
7 & $22.6 \pm 0.7$ & $21.4 \pm 0.7$ \\
8 & $14.0 \pm 0.7$ & $14.2 \pm 0.7$ \\
9 & $10.3 \pm 0.7$ & $8.6 \pm 0.7$ \\
10 & $6.9 \pm 0.7$ & $7.3 \pm 0.7$ \\
11 & $4.5 \pm 0.7$ & $7.6 \pm 0.7$ \\
12 & $13.4 \pm 0.7$ & $12.6 \pm 0.7$ \\
\hline
\end{tabular}

Table 5. Minimum radius of aortic bifurcation (measured at the same $z$ location) using a standard $2 \mathrm{~d}$ method, and working directly on the 3D model.

\begin{tabular}{|c|c|c|}
\hline n. & Angle (grad) & VRML estimation \\
\hline 1 & $139 \pm 12$ & $155 \pm 12$ \\
2 & $127 \pm 12$ & $163 \pm 12$ \\
3 & $128 \pm 12$ & $135 \pm 12$ \\
4 & $145 \pm 12$ & $141 \pm 12$ \\
5 & $135 \pm 12$ & $139 \pm 12$ \\
6 & $117 \pm 12$ & $125 \pm 12$ \\
7 & $124 \pm 12$ & $130 \pm 12$ \\
8 & $140 \pm 12$ & $157 \pm 12$ \\
9 & $131 \pm 12$ & $130 \pm 12$ \\
10 & $136 \pm 12$ & $148 \pm 12$ \\
11 & $139 \pm 12$ & $143 \pm 12$ \\
12 & $132 \pm 12$ & $139 \pm 12$ \\
\hline
\end{tabular}

Table 6. Estimation of intra-aortic angle from 2D slices (left) and from the VRML browser.

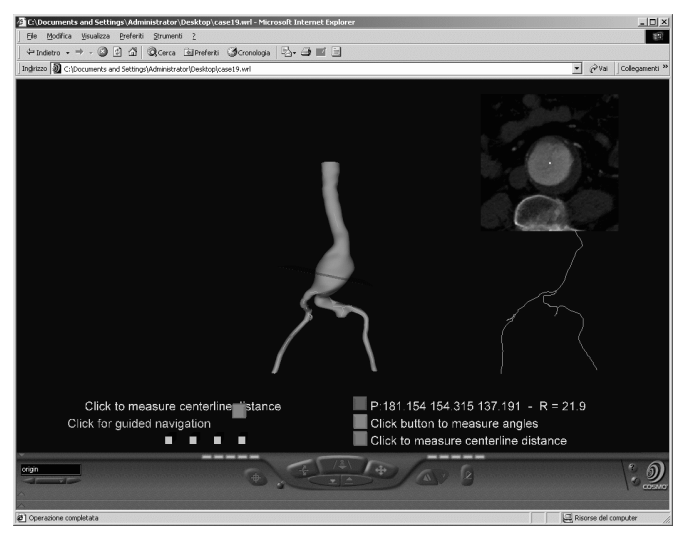

Figure 14. The pre-computed image displayed on the top left represents the reformatted slice (i.e. perpendicular to the vessel centerline), passing near the clicked point.

While the creation of interactive models is something new, the image processing methods are custom versions of well known techniques. We compared the results obtained with different methods, like contour based segmentation, isosurface extraction and 3D simplex balloon inflation. The results suggest that for images acquired using standard protocol, results are equivalent. However, if the quality of images is not perfect it is better to use the more interactive and robust method, like the contour-based, with a strong control by experienced radiologists. Our contour-based approach offers, however, some advantages if compared with other found in literature, for example in [10] where segmentation of contours was used to build tubes, but only on the original MR slices and not on arbitrary slices of the 3D dataset.

The remote analysis of the 3D models seems extremely promising: in fact, the system allows inspections and measurement necessary for the planning of surgical interventions and it is:

- Fast: The VRML measurable models can be published on the net within a few hours from the acquisition, independently from the location of the diagnostic and reconstructing centers. Model download is not usually a problem, being our VRML files sufficiently small: a geometry with 10.000 nodes is stored in a file of about $1 \mathrm{MB}$, that can be gzip compressed to about $300 \mathrm{~K}$, the size of a common image.

- Reliable: almost all the image processing techniques used have been tested in different contexts and applications; the preliminary tests shows also a good accuracy of the measurements if compared with other methods. 
- Easy to use: measuring diameters and distances is just done by clicking with the mouse on a browser window and do not require off line calculations or post processing.

We think also that an approach where the segmentation is performed carefully in a controlled environment can give better results than the use of a simple algorithm as those introduced in the CT consoles by personnel not trained for the specific task.

We plan now to test extensively the reconstruction method and to apply a similar approach to different surgical applications. Colonoscopy is, for example, another field of application where this kind of models can be extremely useful for collaborative study or diagnosis.

In order to have a better validation it will be also necessary to test the measurements results on known phantoms, and w plan to do this in the future. A gallery of demo 3D measurable models is available on our web site (http: //www.crs4.it/ giach).

\section{Acknowledgements}

Special thanks to E. Piccinini, M.D., E. Neri, M.D. for providing data and medical suggestions, and M. Camba for technical help.

\section{References}

[1] G.Abdulaev, S.Cadeddu, G.DeLussu, M.Donizelli, L.Formaggia, A. Giachetti, E.Gobbetti, A. Leone, C. Manzi, P.Pili, A.Scheinine, M. Tuveri, A.Varone, A.Veneziani, G.Zanetti and A.Zorcolo, "ViVa: The Virtual Vascular Project" IEEE trans. on Information Technology in medicine, 14: 134-48 (1998)

[2] J. Piqueras, J.C. and Carreño editors "EuroPacs 1998 Conference proceedings" Barcelona, 1998

[3] S. Laxminarayan editor, "Information Technology Applications in Biomedicine, 2000. Proceedings".

[4] G. Brelstaff, S.Moehrs, P.Anedda,M.Tuveri,G.Zanetti, "Electronic patient records on the Java infobus" In Proceedings of PAJAVA 2000 Conference, Manchester, UK. April 2000

[5] Medical Media Systems http://www.medicalmedia.com

[6] Vital Images - http://www.vitalimages.com

[7] Voxar Plug'n view - http://www.voxar.com
[8] N.W. John, N. Philips, R. Vawda, J. Perrin, "A VRML simulator for Ventricular Catheterisation" in Proceedings of the Eurographics UK conference, Cambridge, UK, April 1999

[9] J.C. Parodi, J.C. Palmaz, H.D. Barone, "Transfemoral intraluminal graft implantation for abdominal aortic aneurysms" Ann Vasc Surg 1991;5:491-499.

[10] J.S. Milner et al. "Hemodynamics of human carotid artery bifurcations: computational studies with models reconstructed from magnetic resonance of normal subjects ." J. of Vascular Surgery, 07/1998, pp.143156

[11] M. Fillinger, "New Imaging Technologies in Endovascular Surgery" Surgical Clinics of North America 79: 3 (1999)

[12] K. Krissian et al. "Model Based Multiscale Detection and Reconstruction of 3D vessels" INRIA Sophie Antipolis Report n.3442 (1998)

[13] A. Kass, A. Witkin and D. Terzopoulos, "Snakes: Active contour models," Int. J. of Comp. Vision 1, 321331 (1988).

[14] L.D. Cohen and I. Cohen, "A finite element method applied to new active contour models and 3D reconstructions from cross-sections" Proc. of 3rd Int. Conf. on Comp. Vision, pp. 587-591 (1990).

[15] H. Delingette, "Simplex meshes: a general representation for 3d shape reconstruction", in CVPR94, pp. 856-859, 1994.

[16] Y. Zhou and A. W. Toga, "Efficient skeletonization of volumetric objects", TVCG, vol. 5, 1999.

[17] K. M. Baskin et al., "Volumetric Analysis of Abdominal Aortic Aneurysm", Medical Imaging 1996: Physiology and Function from Multidimensional Images, Eric A. Hoffman, Editor, Proc. SPIE 2709, p. 323-337 (1996).

[18] J.D. Santilli and S. M. Santilli, "Diagnosis and treatment of Abdominal Aortic Aneurysms" American Family Physician 56:4 (1997)

[19] XOX Corporation - http://www.Xox.com

[20] W.E. Lorensen and H. E. Cline, "Marching cubes: a high resolution 3D surface construction algorithm". In M.C. Stone ed., SIGGRAPH ' 87 Conference Proceedings, pp. 163-170 (1987).

[21] T. Mc Inrey and D. Terzopulos, "Deformable models in medical image analysis, a survey" Medical Image Analysis, 1(2): 840-850, 1996 
[22] D. Magee, A. Bulpitt, E. Berry, “3D Automated Segmentation and Structural Analysis of Vascular Trees Using Deformable Models" Proc. IEEE Workshop on Variational and Level Set Methods in Computer Vision, 2001.

[23] Tillich M, Hill BB, Paik DS, Petz K, Napel S, Zarins CK, Rubin GD., "Prediction of aortoiliac stent-graft length: comparison of measurement methods." Radiology 2001 Aug;220(2):475-83

[24] Sato, Y et al, "Evaluation of intracranial aneurysms with CT angiography: current status and future direction", In SPIE Proceedings Vol. 3033 Medical Imaging 1997: Physiology and Function from Multidimensional Images

[25] Lederle FA, Wilson SE, Johnson GR, Reinke DB, Littooy FN, Acher CW, Messina LM, Ballard DJ, Ansel HJ. Variability in measurement of abdominal aortic aneurysms. Journal of Vascular Surgery 1995; 21:94552. 